TITLE: A systematic review of web-based educational interventions

\title{
Background
}

Web-based educational interventions are used as a medium to promote the exchange of patient education information through online mechanisms that include internet websites (Webb, Joseph, Yardley, Michie, 2010). These educational interventions allow for flexibility in the amount and type of information individuals can access on a routine basis. Typically, individuals are able to access health care related information that can then be used to assist in their overall recovery experience. The educational content is usually based on a comprehensive review of the literature regarding the learning needs of patients following a particular procedure.

Use of web-based information has been shown to be a cost effective means for delivering specialized health care services to patients following hospital discharge (Cote et al., 2011; Martorella, Cote, Racine, Choiniere, 2012; Runge, Lecheler, Horn, Tews, Schaefer, 2006). Specifically, an estimated benefit-cost ratio of 3.65, was identified in terms of incremental morbidity cost savings of $\$ 160,000$ (monetary value reflects direct costs associated with increased number of physician consultations, treatments, and emergency room visits), when the internet was used to deliver patient education interventions (Grace et al., 2006; Runge et al., 2006).

Within the cardiovascular surgical population, self-care education is provided to all patients during their postoperative hospitalization. The intentions of this education are to enhance self-care behaviour performance during the first six months of home recovery. Self-care behaviours following heart surgery are the activities individuals perform to promote their recovery (Fredericks, Lo, Ibrahim, Leung, 2010). These behaviours include 
routine monitoring of fluid and nutrition intake; ongoing assessment and modification of activity performance, such as bathing, dressing, and moving about; the management of new and at times complex drug therapies; and early recognition and response to signs and symptoms of pulmonary, wound, and abdominal complications such as pain, dyspnea, fatigue, and edema (Fredericks, Ibrahim, Puri, 2009).

Self-care patient education interventions are typically presented in the form of written materials. Its content is usually generated from a thorough review of the literature relating to patients' identified learning needs and addresses the following topics: medication, healthy heart diet, activity, signs and symptoms of infection, incision care, and complications (Fredericks, 2009). The effectiveness of standard in-patient education has been examined across the cardiovascular surgical setting (Canadian Hospital Reporting Project, 2010; Fredericks, Ibrahim, Puri, 2009; Fredericks \& DaSilva, 2010; Jaarsma et al., 2000; Moore, 1995). Inconsistent findings have indicated minimal changes related to performance of self-care behaviours (Fredericks, Ibrahim, Puri; Fredericks \& DaSilva; Jaarsma et al.; Moore). The absence of significant findings has been reasoned to be due to the lack of control patients have with regards to the amount and type of content they are able to peruse, and the actual time the materials can be reviewed.

Contrary to standard patient education interventions, evidence suggests patients who have had heart surgery and received educational materials using web-based educational interventions reported better outcomes, than patients who received educational interventions delivered using other media (Runge et al., 2006). Specifically, a statistically significant decline $(\mathrm{p}<.05)$ in the number of physician consultations $(-$ $44 \%$ ), emergency room visits (-67\%), and days off school and/or work (-71\%) was 
noted in patients who received education via the internet (Samoocha, Bruinvels, Elbers, Anema, van der Beek, 2010).

While findings demonstrate the effectiveness of web-based patient education interventions on patient outcomes, they fall short of identifying specific intervention characteristics that are associated with desired outcomes. In particular, the approach (standardized vs. individualized) that is used to design the web-based education and the frequency and length of time in which the content is accessed in relation to enhanced self-care behaviour performance is not known.

\section{Study Purpose}

The purpose of this systematic review was to determine the characteristics of web-based cardiovascular surgical patient education interventions that are associated with producing enhanced performance of self-care behaviours. Specifically, the approach, individualized or standardized, that is most effective for providing web-based patient education interventions; and the frequency and duration of access of the web-based patient education interventions during the postoperative recovery period will be examined.

\section{Method}

\section{Design}

A systematic review of studies to determine the characteristics of web based patient education interventions associated with producing changes in self-care behaviours during the home recovery period was conducted. The PRISMA framework was used to ensure accurate and complete conduct and reporting of this systematic review (Figure 1) (Prisma Statement, 2009). Specifically, areas that relate to the study sample, data 
abstraction, measurement of outcome, data synthesis, and data analysis are discussed below.

Quality assessments were conducted on all studies that were considered eligible for inclusion in this systematic review. The Effective Public Health Practice Project (EPHPP) Quality Assessment Tool (Armijo-Olivio, Stiles, Hagen, Biondo, Cummings, 2010) was used to examine the overall methodological rigour of the study design. The EPHPP included six domains that addressed selection bias, study design, confounders, blinding, data collection method, and withdrawals/ drop-outs. Adequate content and construct validity have been reported. The total scale score was calculated by summing the scores across items and ranged from 1 to 3 . Lower scores suggests higher methodological quality.

\section{Sample}

\section{$\underline{\text { Search strategies }}$}

The search for relevant studies was performed using the following databases: CINAHL, MEDLINE, PUBMED, EMBASE, the Cochrane Methodological Register, Cochrane Database of Systematic Reviews, PsycInfo, Social Sciences Abstracts, ERIC, and HEALTH STAR. The keywords used in the search included: recovery phase, postoperative, coronary artery bypass graft (CABG), valve/valvular replacement (VR), heart surgery, post-surgical, postoperative behaviours, web-based interventions, self-care, self-care behaviour. Following initial searches, the results were combined with the operands AND and OR. Reference lists of studies retrieved were examined for additional studies that address the postoperative recovery period of patients who have had heart surgery. A total of 352 articles were found to have addressed web-based patient 
education. Seventy-two were duplications, which were removed. An additional 261

articles were excluded because the reported study assessed the effectiveness of a combined pre and post web-based educational interventions and non-surgical web-based patient education interventions. A total of 19 studies met the selection criteria and were included in the systematic review (articles included in systematic review are indicated in Reference list with an asterisk).

\section{Study selection: Inclusion Criteria}

Studies were included in the systematic review if they met the following selection criteria: 1$)$ the sample represented adult ( $\geq 18$ years) patients who underwent heart surgery, 2) the outcomes assessed relate to number and type of self-care behaviours performed during the post-hospital discharge recovery period, and 3) the study report was

published in English between 2000 and 2012 and used non-experimental, experimental or randomized clinical trial (RCT), quasi-experimental, mixed methods, and qualitative designs.

\section{Study selection: Exclusion Criteria}

Studies whose participants underwent heart transplants or have a ventricular assist device (VAD) were excluded from this study as these individuals would have received education plus support in the form of routine psychological counseling, home care, and social work visits during their home discharge period (Wrightson 2002). Thus, these patients received a number of psychosocial and educational interventions that are interrelated, during the first few weeks of their home recovery (Wrightson 2002). The findings from studies whose participants underwent heart transplants or VAD will confound the effects of this review, as it may not be possible to isolate the effects of 
patient education interventions delivered to this group. As well, studies in which the participants underwent emergency surgeries were excluded as these individuals will have different postoperative self-care behaviour demands than study participants who underwent elective heart surgeries.

\section{$\underline{\text { Screening }}$}

Studies rather than reports were considered as the unit of interest for this systematic review. The reports were compared based on the names of the authors, location and setting, specific details of the intervention (i.e. the approach -individualized or standardized; and the frequency and duration of access of the web-based patient education interventions during the postoperative recovery period), the number of participants and baseline data, and the date and duration of the study. Thus, multiple reports of the same study were identified and linked together. If uncertainties remained after considering these factors, the authors of the reports were contacted.

\section{Data Abstraction}

The research team extracted data from all articles screened (Table 1). Inter-rater reliability was assessed to demonstrate consistency in data extraction. This was accomplished by two members of the research team who were familiar with the data abstraction process. They independently abstracted data from $10 \%$ of the articles retrieved and then compared results to determine the degree to which they are equivalent. A value of 0.80 or greater was considered an acceptable value of inter-rater reliability, as this is a commonly accepted value in nursing research (Fredericks \& DaSilva, 2011). The inter-rater reliability score of 0.93 was obtained.

\section{Measurement of outcome}


The following information was gathered about each study: year of publication, country in which the study was conducted, study design (non-experimental, quasiexperimental, experimental, qualitative, mixed-method), sample size (total, and for each study group, if relevant), sample demographics (age, sex, marital status, co-morbid condition, type of surgery), drop-out rate, and number and type of study groups (control or comparison and treatment, or two treatment groups). Web-based interventions were characterized in terms of approach (standardized, individualized), frequency of intervention delivery and length of time of delivery. With regards to self-care behaviour performed, the type and number of self-care behaviour was extracted. These data was used for descriptive purposes.

\section{Analyses}

Descriptive statistics were conducted to 1) delineate the characteristics of the studies included in this systematic review, 2) describe the characteristics of individuals comprising the samples, and 3) describe the approach, frequency and length of time engaged with the web-based interventions associated with statistically significant changes in self-care behaviour performance during the post-discharge recovery period. An independent sample t-test was conducted to determine whether or not a statistically significant difference in number of self-care behaviours performed exists between individuals who received a web-based educational intervention and those who received standardized patient education. T-test were conducted using the calculated effect size obtained from outcomes. Effect sizes were calculated using the following formula: Difference between two means divided by a standard deviation of the data.

\section{Findings}




\section{Study characteristics}

The 19 studies that met the inclusion criteria for this systematic review included 1717 participants. Fourteen $(73.7 \%)$ of the studies were of low methodological quality, with the remaining $5(26.3 \%)$ being of moderate quality. Approximately, $70 \%$ of the studies used a convenience sample. Half (50\%) of the studies used an experimental design to evaluate the effectiveness of web-based cardiovascular surgical educational interventions, while $31.6 \%$ of the studies used a mixed-method design. The most frequent method of assignment was randomization (65\%). However, in $60 \%$ of these studies, the type of randomization used was not described. When randomization techniques were described, the most commonly used approach was that of randomization lists (25\%). Three (40\%) occasions of measurement were normally used to collect study data. The average retention rate was $88.8 \%$ (Table 1$)$.

\section{Characteristics of the participants}

The participants in the control group were on average $62.4(\mathrm{SD}=63.1)$ years of age, male (63.1\%), Caucasian (25\%), with a high school (20\%) education. Similarly, the experimental group was on average $63.3(\mathrm{SD}=62.9)$ years of age, male $(62.4 \%)$, Caucasian $(27 \%)$, with a high school (25\%) education. Approximately a quarter $(23.3 \%)$ of the study participants did not have reasonable access to internet due to: 1) poor high speed connections, 2) need to share computer/laptop/ipad with members of household, or 3) not being able to afford electronic resources. Over half (53.3\%) of the studies did not collect data on culture, while none of the studies collected information on socioeconomic or employment status. No statistically significant differences between the groups were noted on demographics characteristics. The study participants had on 
average 5 co-morbid conditions (55\%), with diabetes (84.2\%), high blood pressure (78.9 $\%)$, and high cholesterol (73.6\%) being the most commonly reported co-morbid conditions (Table 1).

Characteristics of education received by control group participants

Generally the control group received a structured educational brochure (74\%) that served as standard of care. This booklet was provided within 24-48 hours of admission to the recovery unit $(94.7 \%)$. The booklet typically addressed the following topics: medication management, nutrition, activity, signs and symptoms of infection, incision care, and complications. Patients were expected to independently review the booklet during their hospitalization. If they required additional information or had any questions, they were instructed to ask their nurse prior to their hospital discharge. In the one instance when the education was delivered post-hospital discharge, it was delivered within the first 24 hours of home delivery, by telephone. The length of time for teaching in the control group was 44.90 minutes ( $\mathrm{SD}=74.5$ minutes) (Table 1).

On average, the control group performed approximately $2.8(\mathrm{SD}=1.6)$ self-care behaviours during their home recovery period. The most common behaviours performed were: taking prescribed medication (95.4\%), preparing meals (89.6\%), and engaging in light activity (82.0\%) (Table 1).

Characteristics of education received by experimental group participants

Type of web-based education: structured vs. tailored

The majority of studies reported the use of a structured web-based patient education (84.2\%) intervention that consisted of pre-designed screens. This type of webbased education required the study participant to review each screen, in sequential order, 
prior to moving to the next educational screen. The content areas addressed throughout the structured web-based education were similar to those presented in the education brochure received by the control group study participants. The findings from studies that examined the effectiveness of structured web-based education were variable, with the majority $(75.0 \%)$ indicating minimal effectiveness of this type of intervention (Table 1).

Three studies examined the effectiveness of a tailored web-based education intervention in which study participants had the option to select their topic area of interest. Thus, they were able to select the topics that they deemed relevant for their specific learning at a particular point in time. They were not required to review all of the educational content in sequential order, but could pre-select specific content areas from various drop-down menus. The findings from these studies indicated statistically significant increases in self-care behaviour performance $(\mathrm{p}<.05)$. The content presented in the tailored web-based education program was similar to the content areas identified in the structured web-based education programs (Table 1).

\section{$\underline{\text { Provision of resources }}$}

Approximately three quarters $(75.0 \%)$ of the studies that examined the effectiveness of web-based patient education interventions required study participants to provide their own equipment (55\%) which consisted of a laptop (35\%), heart monitor (25\%), and/or access to internet (75\%). In cases, where access to the internet and provision of electronic devices was not the responsibility of the study participant $(25 \%)$, internet and access to computers was provided by the study team (Table 1). Type and number of self-care behaviours performed 
Individuals who received a web-based patient education intervention performed approximately $4.0(\mathrm{SD}=.9)$ self-care behaviours during their home recovery period. The most common behaviours performed were: taking prescribed medication (98.2\%), cleaning sternal wound incisions (95.1\%), and engaging in light physical activity (87.3 $\%)$. A statistically significant difference between individuals who received the webbased intervention and those who received standard patient education (i.e. structured educational brochure) in terms of number of self-care behaviours performed during postdischarge recovery is noted: $\mathrm{t}(17)=2.52, \mathrm{p}=.03($ Table 1$)$.

Web-based training

For the most part (52.6\%), training was provided to study participants prior to their involvement in the online education system. This training varied and consisted of one-on-one training sessions between study staff and study participant; provision of a training booklet; or viewing a 10 minutes video that focused on how to use a computer and internet. In all instances, the training occurred during the individual's hospitalization and addressed the following areas: how to turn on/off a computer, becoming familiar with the keyboard, use of a mouse, how to access the internet, and where to find help (Table 1).

\section{Elements of web-based patient education websites}

Even though all of the online education websites provided patient education; how they were set up and the type of additional information that they contained varied. For example, more than half $(60 \%)$ of the websites contained information related to additional resources and references, $40 \%$ provided their study participants with access to an ask an expert chat forum, $30 \%$ created online discussion groups, and approximately 
$10 \%$ had an online skills workshops that could be accessed upon review of the education. Approximately $89 \%$ of the studies contained online intake forms for data collection embedded within the website (Table 1).

For the most part, the majority $(75 \%)$ of the web-based patient education sites were protected. Approximately $45 \%$ of the sites provided study participants with the option to access help, while $30 \%$ of the interventions assessed provided a phone number and $22.2 \%$ supplied an email address for study participants to use should they require help in navigating through the online system (Table 1).

The length of time for access to web-based interventions averaged 503.40 minutes ( $\mathrm{SD}=233.1$ minutes), while the frequency with which individuals accessed the website were variable and ranged between two and 12 times. The studies in which participants engaged in the web-based education intervention over a longer ( $>60$ minutes) period of time $(36.8 \%)$ and accessed the site more than 3 times $(47.3 \%)$ reported statistically significant increases in self-care behaviour performance $(\mathrm{p}<.05)($ Table 1$)$.

\section{Discussion}

Studies included in this systematic review that examined the effectiveness of structured web-based patient education interventions were of low to moderate quality. Thus, further examination of the effectiveness of web-based interventions is needed using more rigorous designs. For the most part, these interventions were standardized and did not allow individuals to skip or bypass information screens. Inconsistent findings were noted. This result is similar to what has been identified in the theoretical literature addressing patient education (Redman, 2001). Study participants were required to view all screens throughout the web-based program. Thus, all patients receive the same 
information related to performance of self-care behaviours, regardless of its relevance. This is problematic as individuals may not be accustomed to going through large volumes of information in a single sitting; may not have the time to review the website; and/or may not be well enough to browse through the various screens.

Conversely, three studies examined the effectiveness of individualized web-based patient education programs. Consistently, these studies reported statistically significant findings related to the effectiveness of the web-based patient education intervention in enhancing performance of self-care behaviours and tended to be of low to moderate quality. These findings are similar to results reported across the literature that has examined the effectiveness of paper-based (for example: booklets, flyers, pamphlets) individualized patient education interventions (Fredericks, Ibrahim, Puri, 2009). Theoretically, the individualized component of the patient education intervention allows the individual to select information that is of relevance to them and their learning at a particular point in time. In doing so, it increases the likelihood that they will be engaged and adheres to the recommendations that are being put forward (Redman, 2001). As well, individuals were not required to peruse all of the information in a single sitting. They had the option to go back to various screens at multiple points in time.

Approximately a quarter of the study participants did not have reasonable access to a computer and/or internet. In all of these cases, internet access and/or computer devices were provided to study participants. The need to either provide internet access or establish a system in which computer hardware can be issued to patients without reasonable access should be considered should web-based interventions be an established part of the post-discharge post-surgical recovery process. 
Web-based interventions will need to be designed to reflect the cultural and socioeconomic factors of individuals, in order to promote its active engagement. However, information on these two demographic characteristics was not investigated. An individual's culture and socioeconomic status will not only provide a clearer description of the sample and thus the population of interest; but may serve to explain findings from studies that investigate the effectiveness of web-based interventions.

Findings indicate during the recovery period, study participants spend just over five hours in total, engaged in the web-based patient education intervention, while 1.2 hours are spent reviewing the patient education booklet. The significant increase in time spent reviewing the web-based education may be a result of the interactive nature of online education, which may make it more enticing for patients to want to continue to review the content. As well, web-based patient education can allow for graphics and text to be animated, imbed the element of sound and/or narration, and provides access to a significant volume of information in a highly organized manner; thus, enticing individuals to remain engaged with the materials.

\section{Application}

Even though findings suggest, using an individualized web-based patient education intervention may be more effective than a booklet or standardized patient education website; adapting these results to the clinical environment may not be feasible in the immediate future, as the site would need to be created, while staff would need to become familiar with navigating the site, as well as its content.

However, incremental changes in how patient education interventions are designed and delivered should be created to allow for the development and 
implementation of an individualized web-based patient education intervention to occur over time. It is suggested that nurses consider integrating other forms of education into their daily care routines. For example, engage patients in conversations related to their home discharge during care related activities (bathing, feeding, changing dressings). That is, ask patients if they have any questions about how to take care of themselves when they go home. The nurse can use prompts such as: do you have any questions about how you should be cleaning your incisions, bathing, or taking your medications when you go home?, then respond to the patient according to their specific questions. In doing so, the nurse is beginning to individualize the amount and type of patient education that is being provided to individuals. This should suffice as a temporary measure until a web-based patient education intervention is designed and implemented.

In order to designing and implementing a web-based patient education intervention, the nurse on the unit will need to work with their organization's information technology department to physically create the website. As the average age of individuals who undergo heart surgery is around the mid to late 60s, the website to be developed should be created with this particular population in mind. In particular, as these individuals are developmentally characterized as elderly, they may be more prone to the development of functional limitations that may include visual, hearing, and cognitive impairments; and reduced hand and motor coordination. Thus, a website that contains a large, clear font size (Arial 16), high contrast lighting to allow for text to stand out, links that are distinguishable from texts, readable language, consistent layouts across the various screens to help with recognition and facilitate ease of navigation, use of a mouse or keyboard to select links, and a talking browser component can be incorporated into the 
design of the web-based patient education intervention (Fredericks, Martorella, Swart, Neuman, in press). A training session that focuses on the basics of using a computer as well as an introduction to the web-based patient education intervention should be designed. This training session can occur as part of a one-on-one teaching session delivered prior to the patient's hospital discharge to familiarize them with the education website.

\section{Conclusion}

Use of web-based information has been shown to be a cost effective means for delivering specialized health care services to patients following hospital discharge. However, the approach used to design the web-based education and the frequency and length of time in which the content is accessed in relation to enhanced self-care behaviour performance is not known. The purpose of this systematic review was to determine the characteristics of web-based cardiovascular surgical patient education interventions that are associated with producing changes in self-care behaviours. Findings indicate the most effective form of web-based patient education is one that is interactive and allows patients to navigate the online system on their own. Specific strategies related to the specific elements nurses should consider as they design individualized web-based

education interventions were provided. However, nurses should be cautious in applying the findings from this systematic review to practice, as studies included were of low to moderate quality. Thus, further examination of the effectiveness of web-based interventions using rigorous designs is need. 


\section{References}

* denotes studies included in systematic review

Armijo-Olivo, S., Stiles, C. R., Hagen, N. A., Biondo, P. D. \& Cummings, G. G. (2012). Assessment of study quality for systematic reviews: a comparison of the Cochrane Collaboration Risk of Bias Tool and the Effective Public Health Practice Project Quality Assessment Tool: methodological research. Journal of Evaluation in Clinical Practice, 18(1), 12-18.

* Artinian, N.T., Harden, J.K., Kronenberg, M.W., Vander Wal, J.S., Daher, E., Stephens, Q., \& Bazzi, R.I. (2003). Pilot Study of a Web-based compliance monitoring device for patients with congestive heart failure. Heart \& Lung, 32(4), 226-233. Doi: 10.1016/S0147-9563(03)00026-8

* Barnason, S., Zimmerman, L., \& Hertzog, M. (2005). Impact of a telehealth intervention to augment home health care on functional and recovery outcomes of elderly patients undergoing coronary artery bypass graft. Heart \& Lung, 35(4), 225-233. Doi: 10.1016/j.hrtlng.2005.10.003

* Barnason, S., Zimmerman, L., Nieveen, J., Schulz, P., Miller, C., Hertzog, M., \& Tu, C. (2009). Influence of a symptom management telehealth intervention on older adults' early recovery outcomes after coronary artery bypass surgery. Heart \& Lung, 38(5), 364-376. Doi: 10.1016/j.hrtlng.2009.01.005

* Barnason, S., Zimmerman, L., Schulz, P., \& Tu, C. (2009). Influence of an early recovery telehealth intervention on physical activity and functioning after coronary artery bypass surgery among older adults with high disease burden. Heart \& Lung, 38(6), 459-468. Doi: 10.1016/j.hrtlng.2009.01.010 
* Bentar, D., Bondmass, M., Ghitelman, J., \& Avitall, B. (2003). Outcomes of Chronic Heart Failure. Archives of Internal Medicine (JAMA), 163, 347-352.

* Brennan, P.F., Casper, G.R., Burke, L.J., Johnson, K.A., Brown, R., Valdez, R.S., Sebern, M., Perez, O.A., \& Stuergeon, B. (2010). Technology-Enhanced Practice for Patients with Chronic Cardiac Disease: Home Implementation and Evaluation. Heart \& Lung, 39(6S), S34-S46. Doi: 10.1016/j.hrtlng.2010.09.003

Canadian hospital reporting project. (2010). Canadian Hospital Reporting Project Information Sheet. Retrieved from: http://www.cihi.ca/CIHI-extportal/pdf/internet/CHRP_INFORMATION_SHEET_EN 2010.

Côté, J., Ramirez Garcia, P., Rouleau, G., Saulnier, D., Guéhéneuc, Y-G., Hernandez, A., Godin, G. (2011). A nursing virtual intervention : Real-time support for managing antiretroviral therapy. Computer, Informatics, and Nursing, 29, 1, 43-51.

* Goldberg, L.R., Piette, J.D., Walsh, M.N., Frank, T.A., Jaski, B.E., Smith, A.L., Rodriguez, R., Mancini, D.M., Hopton, L.A., Orav, J.E., \& Loh, E. (2003). Randomized trial of a daily electronic home monitoring system in patients with advanced heart failure: The Weight Monitoring in Heart Failure (WHARF) trial. American Heart Journal, 146(4), 705-712. Doi: 10.1016/S0002-8703(03)00393-4

Grace, S. L., Krepostman, S., Abramson, B. L., Scholey, P., Brooks, D., Jaglal, S. (2006). Referral to and discharge from cardiac rehabilitation: key informant views on continuity of care. Journal of Evaluation in Clinical Practice, 12,155-163.

* Dew, M.A., Goycoolea, J.M., Harris, R.C., Lee, A., Zomak, R., Dunbar-Jacob, J., Rotondi, A., Griffith, B.P. \& Kormos R.L. (2004). An Internet-Based Intervention to Improve Psychosocial Outcomes in Heart Transplant Recipients and Family 
Caregivers: Development and Evaluation. The Journal of Heart and Lung Transplantation, 23(6), 745-758.

* Delgado D.H., Costigan, J., Wu, R., \& Ross, H.J. (2003). An interactive internet site fort the management of patients with congestive heart failure. Canadian Journal of Cardiology, 19(12), 1381-1385.

Fredericks, S., DaSilva, M. (2011). An examination of the post-discharge recovery experience of patients who have had heart surgery. Journal of Nursing and Healthcare of Chronic Illness, 2, 4, 281-291.

Fredericks, S., Ibrahim, S., Puri, R. (2009). Coronary Artery Bypass Graft Surgery patient education: A systematic review. Progress in Cardiovascular Nursing, $162-168$.

Fredericks, S., Lo, J., Ibrahim, S., \& Leung, J. (2010). An examination of the difference in performance of self-care behaviours between white and non-white patients following CABG surgery: A secondary analysis. Canadian Journal of Cardiovascular Nursing, 20, 4, 21-29.

Fredericks, S., Martorella, G., Swart, B., \& Newman, E. (in press, Oct - Dec 2013). Designing a cardiovascular surgical web-based patient education intervention: A discussion paper. Journal of Nursing Science, vol. 31, no. 4.

Jaarsma, T., Halfens, R., Abu-Saad, H., Dracup, K., Diederiks, J., Tan, F. (2000). Selfcare and quality of life in patients with advanced heart failure: The effect of a supportive educational intervention. Heart and Lung, 29, 5, 319-330. 
* Kleinpell, R.M., \& Avitall, B. (2007). Integrating Telehealth as a Strategy for Patient Management After Discharge for Cardiac Surgery. Journal of Cardiovascular Nursing, 22(1), 38-42.

* Lindsay, S., Smith, S., Bellaby, P., \& Baker, R. (2008). Enabling healthy choices: is ICT the highway to health improvement? Health: An Interdiscplinary Journal, 12(3), 313-331. Doi: 10.1177/1363459308090051

* Lindsay, S., Smith, S., Bellaby, P., \& Baker, R. (2009). The Health Impact of Online Heart Disease Support Group: A Comparison of Moderated Versus Unmoderated Support. Health Education Research, 24(4), 646-654. Doi:10.1093/her/cyp001

* Lindsay, S., Smith, S., Bell, F., \& Bellaby, P. (2007). Tackling the digital divide: Exploring the impact of ICT on managing heart conditions in a deprived area. Information, Communication \& Society, 10(1), 95-114. Doi: $10.1080 / 13691180701193127$

* Maric, B., Kaan, A., Araki, Y., Ignaszewski, A. \& Lear, S.A. (2010). The Use of the Internet to Remotely Monitor Patients with Heart Failure. Telemedicine and eHealth, 16(1), 26-33. Doi: 10.1089/tmj.2009.0094

Martorella, G., Cote, J., Racine, M. Choiniere, M. (2012). Web-Based Nursing Intervention for Self-Management of Pain After Cardiac Surgery: Pilot Randomized Controlled Trial. Journal of Medical Internet Research, Retrieved from: http://www.jmir.org/2012/6/e177/?utm_source=dlvr.it\&utm_medium=feed.

Moore, S. (1995). A comparison of women's and men's symptoms during home recovery after coronary artery bypass surgery. Heart and Lung, 24, 6, 495-501. 
Prisma statement. (2009). Prisma 2009 Flow Diagram. Retrieved from: http://www.prisma-statement.org/statement.htm].

Public Health Agency of Canada. (2008). Managing Cardiovascular Disease. Retrieved from: http://www.phac-aspc.gc.ca/cd-mc/cvd-mcv/.

Redman, N. (2001). The Practice of Patient Education. St. Louis: Mosby.

Runge, C., Lecheler, J., Horn, M., Tews, J. T., Schaefer, M. (2006). Outcomes of a Webbased patient education program for asthmatic children and adolescents. Chest, $129,3,581-593$.

Samoocha, D., Bruinvels, D. J., Elbers, N. A., Anema, J. R., van der Beek, A. J. (2010). Effectiveness of web-based interventions on patient empowerment: a systematic review and meta-analysis. Journal of Medical Internet Research, 12, 2, e23.

* Scherrer-Bannerman, A., Fofonoff, D., Minshall, D., Downie, S., Brown, M., Leslie, F., \& McGowan, P. (2000). Web-based education and support for patients on the cardiac surgery waiting list. Journal of Telemedicine and Telecare, 6(2), 72-74.

* Southard, B. H., Southard, D.R. \& Nuckolls, J. (2003). Clinical Trial of an Internetbased Case Management System for Secondary Prevention of Heart Disease. Journal of Cardiopulmonary Rehabilitation, 23(1), 341-348.

Webb, T. L., Joseph, J., Yardley, L., Michie, S. (2010). Using the internet to promote health behavior change: a systematic review and meta-analysis of the impact of theoretical basis, use of behavior change techniques, and mode of delivery on efficacy. Journal of Medical Internet Research, 12, 1, e4.

Wrightson, N., Blake, A., English, L. (2002). Care of the heart transplant patient. Nursing Times, 98, 28, 34-37. 
* Zimmerman, L., Barnason, S., Nieveen, J., \& Schmaderer, M. (2004). Symptom Management Intervention in Elderly Coronary Artery Bypass Graft Patients. Outcomes Management, 8(1), 5-12.

* Zimmerman, L., Barnason, S., Schulz, P., Nieveen, J., Miller, C., Hertzog, M., Rasmussen, D., \& Tu, C. (2007). The Effects of a Symptom Management Intervention on Symptom Evaluation, Physical Functioning, and Physical Activity for Women After Coronary Artery Bypass Surgery. Journal of Cardiovascular Nursing, 22(6), 493-500.

* Zutz, A., Ignaszewski, A., Bates, J. \& Lear, S.A. (2007). Utilization of the Internet to Deliver Rehabilitation at a Distance: A Pilot Study. Telemedicine and e-Health, 13(3), 323-330.

This manuscript was accepted for publication by Sage Publications, Fredericks, S., Martorella, G. \& Catallo, C. (2014). A systematic review of web-based educational interventions. Clinical Nursing Research. 1-23. DOI: 10.1177/1054773814522829 


\section{Notes}

The research team wishes to acknowledge funding received from the Primary Health

Care System (PHCS) Program. This funding has been instrumental in helping the team to move forward with starting this systematic review. 